\title{
Renal colic pain and the TENS as an alternative analgesia
}

\author{
Umadevan Rajasagaram*
}

Emergency Department, Angliss Hospital, Albert Street, Upper Ferntree Gully, VIC 3156, Australia

Kidney stones are prevalent in the "stone belt" region which extends over America (Southeast), Africa (North), Middle East (Southeast) and Australia (Northeast) [1]. Globally, the lifetime prevalence of stone disease is 10 to $15 \%$ and it counts for millions of patient visits to the emergency department (ED), or the outpatient clinics $[1,2]$. The incidence of renal stones is globally increasing with an estimated prevalence ranging up to $15 \%$. During lifetime, approximately $7 \%$ of women and $13 \%$ of men will develop a renal stone. The main aim of management in the Emergency Department is to provide fast, effective and safe analgesia to patients. Most studies published in relation to the acute management of pain in renal colic focuses on the use of prescribed analgesia as described above with little emphasis on the use of other modalities of pain control such as TENS.

The pain associated with renal colic is usually very excruciating with the most commonly prescribed analgesia in renal colic are nonsteroidal anti-inflammatory drugs (NSAIDS), opioids and paracetamol $[3,4]$. Both these drugs have significant side effects with prolonged use. In regard to opioid analgesia prolonged use can lead to opioid addiction as well as in the elderly population can lead to gait instability which can cause significant injuries. The prolonged use of NSAIDs can lead to acute kidney injury as well as upper gastrointestinal bleeds and in regard to paracetamol prolonged use can lead to hepatotoxicity.

TENS has been used to complement traditional analgesia in both labour, postoperative pain as well as musculoskeletal pain $[5,6]$. There has been limited study in the past which has showed that TENS was an effective means of pain control in patients with suspected renal colic delivered by emergency care workers [7]. However, in this study TENS was applied to a variety of patients with symptoms suggestive of renal colic but without confirmation of renal stones in the back of an ambulance. In a world with a growing dependence on prescribed analgesia, TENS offers an alternative as a fast, alternative and effective way of delivering analgesia without the side effects of prescribed analgesia.

Copyright: $(2019$ Rajasagaram U. This is an open-access article distributed under the terms of the Creative Commons Attribution License, which permits unrestricted use, distribution, and reproduction in any medium, provided the original author and source are credited.
TENS is only applied locally over the affected kidney area resulting in minimal systemic side effects that are commonly seen in prescribed pharmaceutical agents. Furthermore, in view of its portability as well as its relative ease of use, TENS can be used in the outpatient setting as well as in the Emergency Department and/or inpatient setting. TENS is already currently being used in an outpatient setting as analgesic option for musculoskeletal pain as well as post-operative pain. Research into other modalities of pain control will help provide a better understanding of the analgesic options available to patients suffering renal colic rather than the sole reliance on pharmaceutical therapy. This hopefully would lead to a decrease in the prescription of pharmaceutical analgesia and the associated side effects associated with it.

\section{References}

1. Fisang C, Anding R, Muller SC, Latz S, Laube N (2015) Urolithiasis-an interdisciplinary diagnostic, therapeutic and secondary preventive challenge. Deutsches Arzteblattinternational 112: 83-91. [Crossref]

2. Ghani KR, Roghmann F, Sammon JD, Trudeau V, Sukumar S, et al. (2014) Emergency department visits in the United States for upper urinary tract stones: trends in hospitalization and charges. J Urol 91: 90-96. [Crossref]

3. Pickard R, Starr K, MacLennan G, Kilonzo M, Lam T, et al (2015) Use of drug therapy in management of symptomatic ureteric stones in hospitalised adults: a multicentre, placebo-controlled, randomised control trial and cost effectiveness analysis of a calcium channel blocker(nifedipine) and an alpha -blocker (Tamsulosin) (the SUSPEND trial). Health Technol Assess 19: 1-171. [Crossref]

4. Afshar K, Jafari S, Marks AJ, Eftekhari A, MacNeily AE (2015) Non-steroidal anti-inflammatory drugs (NSAIDS) and non-opoids for acute renal colic. Cochrane Database Syst Rev 29: CD006027. [Crossref]

5. Dowswell T, Bedwell C, Lavender T, Neilson JP (2009) Transcutaneous electrical nerve stimulation (TENS) for pain management in labour. Cochrane Database Syst Rev 2: CD007214. [Crossref]

6. Bjordal JM, Johnson MI, Ljunggreen AE (2003) Transcutaneous electrical nerve stimulation (TENS) can reduce postoperative e analgesic consumption. A metaanalysis of optimal treatment parameters for postoperative pain. Eur J Pain 7: 181-188. [Crossref]

7. Mora B, Giorni E, Dobrovits M, Barker R, Lang T, et al. (2006) Transcutaneous electrical nerve stimulation: An Effective Treatment for Pain Caused by Renal Colic in Emergency Care. J Urol 175: 1737-1741. [Crossref]
*Correspondence to: Umadevan Rajasagaram, Emergency Department, Angliss Hospital, Albert Street, Upper Ferntree Gully, VIC 3156, Australia, E-mail: Umadevan.Rajasagaram@easternhealth.org.au

Received: June 03, 2019; Accepted: June 13, 2019; Published: June 18, 2019 